\title{
Role of UGT1A1 and ADH gene polymorphisms in pegvisomant-induced liver toxicity in acromegalic patients
}

\author{
M Filopanti ${ }^{1,2, *}$, A M Barbieri ${ }^{1,2, *}$, G Mantovani ${ }^{1,2}$, S Corbetta ${ }^{3}$, V Gasco ${ }^{4}$, \\ M Ragonese ${ }^{5}$, C Martini ${ }^{6}$, F Bogazzi ${ }^{7}$, A Colao ${ }^{8}$, D Ferone ${ }^{9}$, A Peri ${ }^{10}$, F Pigliaru ${ }^{11}$, \\ G Angeletti ${ }^{12}$, M Arosio ${ }^{13}$, P Beck-Peccoz ${ }^{1,2}$, A G Lania ${ }^{14}$ and A Spada ${ }^{1,2}$ \\ ${ }^{1}$ Endocrinology and Diabetology Unit, Fondazione IRCCS Ca' Granda Ospedale Maggiore Policlinico, Padiglione \\ Granelli via F. Sforza 35, 20122 Milan, Italy, ${ }^{2}$ Department of Clinical Sciences and Community Health, University of \\ Milan, Milan, Italy, ${ }^{3}$ Endocrinology and Diabetology Unit, IRCCS Policlinico San Donato, San Donato Milanese, Italy, \\ ${ }^{4}$ Division of Endocrinology, Diabetology, and Metabolism, Department of Internal Medicine, University of Turin, \\ Turin, Italy, ${ }^{5}$ Department of Clinical and Experimental Medicine, University of Messina, Messina, Italy, ${ }^{6}$ Internal \\ Medicine 3, Department of Medicine, University of Padua, Padua, Italy, ${ }^{7}$ Endocrinology Section, Department of \\ Clinical and Experimental Medicine, University of Pisa, Pisa, Italy, ${ }^{8}$ Dipartimento di Medicina Clinica e Chirurgia, \\ Università 'Federico II', Naples, Italy, ${ }^{9}$ Endocrinology, Department of Internal Medicine and Medical Specialties and \\ Center of Excellence for Biomedical Research, IRCCS San Martino-IST, University of Genoa, Genoa, Italy, ${ }^{10}$ Endocrine \\ Unit, Department of Experimental and Clinical Biomedical Sciences, University of Florence, Florence, Italy, \\ ${ }^{11}$ Endocrinology and Diabetology Unit, Azienda Ospedaliero Universitaria of Cagliari, Cagliari, Italy, ${ }^{12}$ Section of \\ Internal Medicine and Endocrinological and Metabolic Sciences, Internal Medicine Department, University of \\ Perugia, Perugia, Italy, ${ }^{13}$ Unit of Endocrine Diseases and Diabetology, Ospedale San Giuseppe Multimedica, \\ Milan, Italy and ${ }^{14}$ Endocrine Unit, IRCCS Humanitas Clinical Institute, Rozzano, Italy \\ *(M Filopanti and A M Barbieri contributed equally to this work)
}

Correspondence should be addressed to A Spada Email anna.spada@unimi.it

\begin{abstract}
Context: Hepatotoxicity is one of the most serious adverse effects in acromegalic patients treated with pegvisomant (PEG-V). Recent studies have found an association between this adverse event and the UGT1A1 allele 28 polymorphism associated with Gilbert's syndrome.

Objective: To determine whether UGT1A1*28 and alcohol dehydrogenase (ADH) polymorphisms influence liver toxicity during PEG-V treatment.

Design and setting: Multicenter observational retrospective study conducted in 13 tertiary care endocrinology units in Italy. Patients: A total of 112 patients with active disease resistant to somatostatin analogs (SSTa) and 108 controls were enrolled. Interventions: Clinical and biochemical data were recorded by electronic clinical reporting forms. Blood or DNA samples were sent to the coordinating center for genotyping.

Results: No differences in genotypes between patients and controls were found. During PEG-V therapy liver function tests (LFT), abnormalities and overt hepatotoxicity developed in 17 and $4.5 \%$ of patients respectively. Logistic and linear regression analyses showed an association between LFT abnormalities during the follow-up visit and prior events of LFT abnormalities in medical history (odds ratio $=1.25 ; P=0.04$ ) and the number of concomitant medications, other than SSTa $(B=3.9 ; P=0.03)$. No correlation between LFT alterations and UGT1A1 allele 28 as well as ADH1C and B polymorphisms was found.

Conclusions: UGT1A1 allele 28 and ADH1C and B polymorphisms do not predict increased risk of hepatotoxicity during PEG-V therapy. Conversely, patients with multi-therapies and with previous episodes of liver disease should be carefully managed, due to the observed association between these conditions and LFT abnormalities during PEG-V therapy.
\end{abstract}




\section{Introduction}

Acromegaly is a chronic disorder due to growth hormone (GH) hypersecretion, the most frequent cause of which is a pituitary adenoma. Persistently elevated serum GH and insulin-like growth factor 1 (IGF1) levels lead to substantial morbidity and mortality. When the first-line therapies, i.e. surgical removal of the pituitary adenoma, administration of somatostatin analogs (SSTa), or dopamine agonists, or radiotherapy, fail to normalize GH and IGF1 levels, pegvisomant (PEG-V) may be effectively used to control the disease (1). PEG-V is an analog of human GH with antagonistic properties due to specific substitution and pegylation (1). PEG-V is efficacious in controlling acromegalic disease in more than $90 \%$ of patients $(2,3,4,5)$. Although PEG-V is generally well tolerated, several adverse effects may lead to treatment discontinuation. In particular, lipohypertrophy at the injection site and rise in the level of liver enzymes identified by liver functional tests (LFT), i.e. glutamic oxaloacetic transaminase (GOT), glutamicpyruvic transaminase (GPT), gamma-glutamyl transpeptidase $(\gamma-G T)$, are the most serious adverse effects (6). Liver injury occurs in $1-8 \%$ of patients. Previous studies have reported an increased risk of liver injury when SSTa are administered in association with PEG-V $(4,5,6)$.

Little is known about the pathogenetic mechanism of PEG-V-induced hepatotoxicity. Idiosyncratic mechanisms have been proposed, as well as biliary obstruction involvement (6).

In a recent study, variants of the uridine diphosphate5'-glucuronosyltransferase type 1A (UGT1A1) gene (7), which codes for proteins involved in the conjugation of different substrates with uridine diphosphoglucuronic acid to facilitate their biliary or renal elimination, have been investigated in acromegalic patients during PEG-V therapy. In particular, the common UGT1A1 allele 28 polymorphism (UGT1A1*28), which is necessary, but not sufficient, for the clinical expression of Gilbert's syndrome (8), was more frequent in acromegalics with hepatotoxicity, thus representing, together with male gender, a predictor of LFT abnormalities (7). Other studies pointed out a role of diabetes mellitus, with significant odds ratios (OR) (from 2 to 5) for the association with PEG-V hepatotoxicity $(9,10)$.

It is unclear whether the polyethylene glycol component of the PEG-V molecule accounts for the induction of adverse effects. Both pure and drug-linked polyethylene glycol clearance is dependent on the molecular weight and it occurs mostly by urinary excretion (11). In addition, biliary excretion and polyethylene glycol metabolism by alcohol dehydrogenase (ADH) have been described in humans (11). As pharmacogenetics studies on normal subjects and on patients with alcohol abuse showed a role of some $\mathrm{ADH} 1 \mathrm{~B}$ and $1 \mathrm{C}$ gene polymorphisms in ethanol pharmacokinetics and hepatopathy $(12,13,14,15,16)$, the aim of this study was to evaluate possible associations between PEG-V-induced liver injury and the previously reported UGT1A1*28 variant and additionally with some $\mathrm{ADH}$ polymorphisms involved in liver disorders or alcohol metabolism.

\section{Subjects and methods}

\section{Patients}

The study was approved by the ethical committee of each center and a written informed consent was obtained from all participants before inclusion. One hundred and twelve patients affected with acromegaly treated with PEG-V were enrolled by 13 Italians centers of endocrinology with the following inclusion criteria: i) age $>18$; ii) Italian drug agency (AIFA) criteria for PEG-V administration fulfilled (uncontrolled disease after trans sphenoidal pituitary adenomectomy, SSTa treatment, and/or pituitary radiotherapy); iii) PEG-V treatment duration more than 3 months; and iv) signed informed consent.

Eighty-seven patients of the study were already included in a previous pharmacogenetics study (17). Complete clinical and hormonal data of the patients are reported in Table 1.

A group of 108 normal subjects were included as the control population for genotypes frequencies.

\section{Genetic analyses}

Genomic DNA was extracted from peripheral blood leukocytes by illustra DNA Extraction Kit BACC2 (GE Healthcare, Little Chalfont Buckinghamshire, UK) according to the manufacturer's instructions. Polymorphisms genotyping of all patients and controls was performed at the same laboratory. The following polymorphisms were analyzed: for UGT1A1 gene UGT1A1*28 polymorphism ([A(TA) 7 TAA] vs WT UGT1A1 [A(TA)6TAA]), for $A D H 1 B$ gene SNPs rs1229984 His48Arg and rs2066702 Arg370Cys, and for $A D H 1 C$ gene SNPs rs1693482 Arg272Gln and rs698 Ile350Val. For genotyping details, see Supplementary Table 1, see section on supplementary data given at the end of this article. All PCRs were performed on an Applied 
Table 1 Basal clinical and biochemical data of a cohort of 112 patients affected with acromegaly and treated with pegvisomant.

\begin{tabular}{lc}
\hline Sex (males \%) & 51.7 \\
Age (years) & $43.2 \pm 14.7$ \\
GPT (U/l) & $20 \pm 11$ \\
GOT (U/l) & $20 \pm 9$ \\
Gamma-glutamyltransferase (U/l) & $15(10.8-22)^{\mathrm{a}}$ \\
Indirect bilirubin (mg/dl) & $0.47(0.34-0.77)^{\mathrm{a}}$ \\
Direct bilirubin (mg/dl) & $0.14(0.1-0.2)^{\mathrm{a}}$ \\
Previous abnormal liver function tests (\%) & 6.3 \\
Alcohol consumption >40 g/day (males) & 2.7 \\
$\quad$ or > 24 g/day (females) (\%) & 39.3 \\
Prior cholelithiasis or (\%) & 7.1 \\
Gilbert's syndrome (\%) & 17.7 \\
Nonalcoholic steatohepatitis (NASH) (\%) & 3.5 \\
Chronic viral hepatitis (HBV or HCV) (\%) & 12.5 \\
Diabetes mellitus (\%) & \\
Therapies & 90.4 \\
Prior SSTa treatment (\%) & $48(22-96)^{\mathrm{a}}$ \\
Prior SSTa treatment duration (years) & 45.5 \\
SSTa coadministration (\%) & $10.9 \pm 5.0$ \\
Initial pegvisomant dosage (mg/day) & \\
\hline
\end{tabular}

$\mathrm{HBV}$, hepatitis B virus; HCV, hepatitis C virus; SSTa, somatostatin analogs. anterquartile range is shown in brackets.

Biosystems 2720 Thermal Cycler, using 0.5 U GoTaq DNA Polymerase (Promega) in a total volume of $25 \mu \mathrm{l}$. Ten microliters of PCR products were digested with $2 \mathrm{U}$ of the appropriate restriction enzyme (New England Biolabs, Ipswich, MA, USA) in a volume of $20 \mu \mathrm{l}$ according to the manufacturer's instructions. About 5\% randomly selected samples underwent direct sequencing to confirm the results of RFLP analysis. Direct sequencing of the PCR products was performed using the BigDye Terminator v3.1 Cycle Sequencing Kit and 3130 Genetic Analyzer (Applied Biosystems).

\section{Study design}

After informed consent, a unique numeric identifier was generated for each patient and linked to his or her clinical data in an electronic clinical reporting form. Patients' blood samples, labeled with respective numeric identifier, were sent to the coordinating center for genetic analysis. Patients' clinical, radiological, and biochemical data were recorded by online case report form both at baseline and for each follow-up visit. Upper normal limits (ULN) were also registered. Hepatological aspects were thoroughly evaluated by reporting the presence of biliary obstruction disease, presence of chronic liver disease, and daily alcohol consumption, prior and during PEG-V treatment. The presence of one or more previously resolved episodes of LFT increase in clinical history as well as diabetes mellitus has been recorded. Gilbert's syndrome was diagnosed by the presence of the following: i) unconjugated hyperbilirubinemia noted on several occasions; ii) normal complete blood count; iii) normal LFT; and iv) no other liver diseases. Finally, PEG-V dosage and other coadministered drugs were recorded.

At the closure of the study, data from monitoring visits were screened by the software, which detected an LFT increase above the corresponding ULN and labeled it as 'mild' if $<3 \times$ ULN or 'overt' if $\geq 3 \times$ ULN. Then, these records were filtered, printed, and grouped for each patient. Finally, they were reviewed by a physician, who checked the following: i) if no gross errors in data insertion were present; ii) clinical and biochemical liver features of the patient before the treatment: iii) if and how the event was managed (treatment modification or withdrawal, radiological examinations, etc.); and iv) what medications were taken.

\section{Statistical analysis}

Hardy-Weinberg equilibrium of genotypes was checked by $\chi^{2}$-test. Correlations with polymorphisms were studied by both considering the three groups (two of homozygotes and one of heterozygotes) and two groups (carriers of the less frequent allele or not) of genotypes, as in previous studies $(17,18)$. Data were reported as mean \pm s.D. when normally distributed or as median and 25-75 percentile range when the distribution was non-Gaussian. Two groups-comparisons were evaluated by Student's t-test whereas three groups were evaluated by ANOVA (followed by Bonferroni post-test). Equality of variances was checked using Levene's test. Nominal data were analyzed by Fisher's exact test in $2 \times 2$ contingency tables or $\chi^{2}$-test, while the other non-Gaussian variables were evaluated by Mann-Whitney $U$ test. Backward linear or logistic multivariate regression analyses were performed with stepwise method, using $P>0.10$ as removal criterion. Two-tailed $P$ values were considered statistically significant when $<0.05$. In multiple comparisons, $P$ value threshold for statistical significance was reduced after Bonferroni correction. Calculations were made by IBM SPSS Statistics, Version 20.0.

\section{Results}

\section{Baseline clinical characteristics and genotypes}

After online case report analysis, 112 patients $(51.7 \%$ males, $43 \pm 15$ years) with complete clinical, biochemical, 
and pharmacological data were included in the study (Table 1). All patients were treated with $10.9 \pm 5.0 \mathrm{mg} /$ day of PEG-V as initial dosage, and 51 patients (45.5\%) also received SSTa. The median follow-up duration was 17 months (interquartile range (IQR): 7-22, range 7-22). Twenty-one patients had 6 months' follow-up duration, while $28.6 \%$ of patients had a follow-up $\leq 12$ months.

Gilbert's syndrome was present in $7.1 \%$ of patients. Hepatitis (nonalcoholic fatty liver disease or chronic viral hepatitis) was detected in $21.2 \%$ of acromegalics. The prevalence of high alcohol consumption (more than $40 \mathrm{~g}$ /day in males or $24 \mathrm{~g} /$ day in females) in this population was $2.7 \%$. Forty-four patients (39.3\%) had current or past cholelithiasis or gallbladder sludge (biliary sand). An elevation of LFT in clinical history has been reported in $6.3 \%$ of patients. Finally, the median number of concurrent medical treatments was 3 (IQR: 1-5).

As far as UGT1A1 polymorphism was concerned, $54.5 \%$ of patients were allele 28 carriers $(8.1 \%$ UGT1A1*28 homozygotes), with no significant difference compared with controls (Table 2). Due to the small number of subjects who were homozygous for the UGT1A $1 * 28$ polymorphism, the patients were grouped as UGT1A1*28 (homo- and heterozygous) and WT carriers for the purpose of statistical analysis.

Those who were homozygous for ADH1B rs2066702 $\mathrm{C} / \mathrm{T}$ polymorphism showed low variability: $\mathrm{C} / \mathrm{C}$ homozygotes were $98.2 \%$ in acromegalics and $100 \%$ in controls. ADH1B rs1229984 G/A polymorphism analysis identified

Table 2 UGT1A1, ADH1C, and ADH1B polymorphisms frequencies (percentage of genotypes) in 112 acromegalics and 108 normal subjects.

\begin{tabular}{lllll}
\hline UGT1A1 allele 28 & $28-I-$ & $28+I-$ & $28+I+$ & $P$ \\
Patients & 45.5 & 46.4 & 8.1 & \\
Controls & 43.5 & 40.7 & 15.7 & 0.26 \\
ADH1B rs1229984 & G/G & G/A & A/A & \\
Patients & 75.0 & 21.4 & 3.6 & \\
Controls & 87.0 & 13.0 & 0.0 & $0.03^{\mathrm{a}}$ \\
ADH1B rs2066702 & $\mathrm{C} / \mathrm{C}$ & $\mathrm{C} / \mathrm{T}$ & $\mathrm{T} / \mathrm{T}$ & \\
Patients & 98.2 & 1.8 & 0.0 & \\
Controls & 100.0 & 0.0 & 0.0 & $1.00^{\mathrm{b}}$ \\
ADH1C rs1693482 & $\mathrm{G} / \mathrm{G}$ & $\mathrm{G} / \mathrm{A}$ & $\mathrm{A} / \mathrm{A}$ & \\
Patients & 62.3 & 36.6 & 0.9 & \\
Controls & 51.9 & 48.1 & 0.0 & $0.14^{\mathrm{b}}$ \\
ADH1C rs698 & $\mathrm{A} / \mathrm{A}$ & $\mathrm{A} / \mathrm{G}$ & $\mathrm{G} / \mathrm{G}$ & \\
Patients & 63.4 & 29.5 & 7.1 & \\
Controls & 50.9 & 41.7 & 7.4 & 0.15 \\
\hline
\end{tabular}

${ }^{a} P<0.05$ but above Bonferroni's correction for multiple comparison tests $(\alpha=0.0102)$

${ }^{b}$ Fisher's exact test on first vs second and third columns grouped. a higher proportion of allele A carriers in acromegalic patients compared with the normal population $(25.0 \%$ vs 13.0, $P=0.0267$ ), but above the Bonferroni correction for multiple comparisons. ADH1C rs1693482 G/A and rs698 A/G allele frequencies were similar to those observed in the normal population (Table 2). In this cohort of patients, all the polymorphisms analyzed were in HardyWeinberg equilibrium.

At baseline, UGT1A1*28 allele was associated with Gilbert's syndrome $(P<0.001)$ as well as with higher bilirubin levels $(P=0.002)$. No significant associations between ADH1C rs1693482, ADH1C rs698, ADH1B rs1229984, and ADH1B rs2066702 polymorphisms and LFT values were found (data not shown).

\section{Abnormal liver function tests during PEG-V treatment and genotypes}

Overt hepatotoxicity, i.e. LFT above threefold ULN, was observed during PEG-V treatment in five patients (4.5\%, three males) administered with PEG-V with a mean dosage of $20 \mathrm{mg} /$ day (Table 3). Hepatotoxicity occurred within 2-3 years (range 2-31 months) after the start of the treatment (10-25 mg/day), and after 2-5 months LFT decreased below 3-ULN with a persistent slight transaminases elevation. Of these patients with overt hepatotoxicity, four had previously diagnosed gallstones, one with an episode of biliary colic a week before the follow-up visit, and three had evidence of previous LFT abnormalities in clinical history. Two patients were in PEG-V plus SSTa administration. Two patients (one of them was diabetic, treated with oral hypoglycemic agents plus glargine) took 12-13 per os medications. All five patients were administered with at least one well-known hepatotoxic drug. In particular, statins in two patients, acetaminophen or other non-steroidal anti-inflammatory drugs in three patients, testosterone esters in two patients, allopurinol in one patient, and iron supplementation in one patient. One patient had hepatotoxicity after oxcarbazepine start. Only one patient needed PEG-V withdrawal and hospitalization due to severe cholestasis. One patient spontaneously reduced PEG-V dosage to $15 \mathrm{mg}$ every other day. No particular alleles frequencies were observed in this group of patients (Table 3 ).

Fourteen patients (12.5\%) experienced a mild hepatotoxicity, i.e. LFT increase less than 3-ULN, within 3 months of treatment, which did not require PEG-V discontinuation (Table 3). PEG-V dosage was $24 \pm 10 \mathrm{mg}$ /day. LFT normalization was achieved after 2-8 months in half of the patients. Of patients with mild 
Table 3 Characteristics and genotype of 112 acromegalic patients who developed or not abnormal LFT during pegvisomant treatment.

\begin{tabular}{|c|c|c|c|}
\hline & Normal LFT $n=93$ & Mild hepatotoxicity $n=14$ & Overt hepatotoxicity $n=5$ \\
\hline Males (\%) & 50.5 & 50.0 & 60.0 \\
\hline PEG-V dosage $(\mathrm{mg} / \text { day })^{a}$ & $19.0 \pm 10.5$ & $24.0 \pm 9.7$ & $20.0 \pm 11.0$ \\
\hline $\begin{array}{l}\text { Median (IQR) number of concomitant } \\
\text { medications }{ }^{\text {b }}\end{array}$ & $3(1-6)$ & $3(2-6)$ & $7(5-12)$ \\
\hline Cholelithiasis (\%) & 38.7 & 29.0 & 80.0 \\
\hline Previous LFT increase $(\%)^{\mathrm{b}}$ & 4.3 & 14.3 & 60.0 \\
\hline Gilbert's syndrome (\%) & 7.5 & 7.1 & 0.0 \\
\hline SSTa coadministration (\%) & 40.9 & 57.1 & 40.0 \\
\hline Diabetes mellitus $(\%)^{\mathrm{b}}$ & 11.8 & 14.3 & 20.0 \\
\hline \multicolumn{4}{|l|}{ Genotype } \\
\hline UGT1A1 (allele 28\%) & 54.8 & 50.0 & 40.0 \\
\hline ADH1B rs1229984 (allele A \%) & 24.7 & 28.6 & 20.0 \\
\hline ADH1B rs2066702 (allele T \%) & 2.1 & 0.0 & 0.0 \\
\hline ADH1C rs1693482 (allele A \%) & 37.6 & 35.7 & 20.0 \\
\hline ADH1C rs698 (allele G \%) & 36.6 & 28.6 & 20.0 \\
\hline
\end{tabular}

LFT, liver function tests; IQR, interquartile range; SSTa, somatostatin analogs; PEG-V, pegvisomant. Mild hepatotoxicity: LFT increase between upper normal limit (ULN) and 3-ULN. Overt hepatotoxicity: LFT increase over 3-ULN.

${ }^{a}$ Normal LFT group receiving average PEG-V dosage at last visit is reported. In mild and overt hepatotoxicity groups, the mean PEG-V dosage at the episodes of liver toxicity are reported.

${ }^{\mathrm{b}}$ Number of concomitant medications, known history of LFT increase, and diabetes mellitus were significant predictors of hepatotoxicity after logistic and linear regression analysis (see Results).

hepatotoxicity, two patients (14.3\%) had previous episodes of LFT abnormalities in clinical history, four (29\%) had gallstones, and two were diabetic (treated with oral hypoglycemic agents). Eight patients (52.7\%) were administered with SSTa. In this group of patients, the median number of concomitant medications was 3 (IQR: 2-6). Statins were administered in $35 \%$ of patients. Fourteen percent of patients took acetaminophen (one of this patient frequently used nimesulide). One patient was administered with testosterone esters, and one with carbamazepin.

In the group of patients with mild hepatotoxicity, UGT1A1 and ADH1B or 1C genotypes were not significantly different from those of the remaining patients (Table 3).

No striking differences between mild and overt hepatotoxicity groups were seen, although a trend in the proportion of previous events of LFT increase, cholelithiasis, as well as in number of concomitant medications, among groups, was observed (Table 3). As regards to SSTa coadministration, this was similar in the groups (Table 3) and the overall rate of hepatotoxicity in patients receiving SSTa plus PEG-V therapy was not significantly different to that observed in patients under PEG-V monotherapy (20.1 vs $14.0 \%, P=0.47$ ).

In order to evaluate the effect of genotypes together with other confounders, such as sex, PEG-V dosage, diabetes mellitus, alcohol consumption, biliary obstruction, hepatopathy, and concomitant medications (in particular SSTa), stepwise multivariate regression analyses were carried out. The dichotomous event 'an increase above ULN of one or more LFT in at least one monitoring visit' was considered the dependent variable of a backward stepwise logistic regression analysis, using the following factors and covariates: male sex, high daily alcohol consumption, biliary tract obstruction (gallstones, gallbladder surgery), presence of prior LFT increase in clinical history, chronic hepatitis (viral, metabolic, nonalcoholic liver steatosis), PEG-V dosage, SSTa coadministration, number of other concomitant medications, and finally UGT1A1, ADH1B, and ADH1C alleles. The only factor retained in the final model was prior LFT increase $(\mathrm{OR}=1.25,95 \%$ CI 1.09-1.45, $P=0.04)$. SSTa coadministration and male sex were not associated with increased risk of hepatotoxicity $(\mathrm{OR}=0.79,95 \% \mathrm{CI} 0.24$ 2.56, $P=\mathrm{NS}, \mathrm{OR}=0.72,95 \% \mathrm{CI} 0.21-2.5, P=\mathrm{NS}$, respectively). No UGT1A1 or ADH genes polymorphisms have been retained in the model (Fig. 1). This logistic regression model was still statistically significant when applied to only GOT or GPT increase events, while it was not significant in case of gamma-GT elevations (data not shown). The same predictors were included in three independent multivariate linear regression analyses, whose dependent variables were GPT, GOT, and gamma-GT highest level recorded during follow-up visits. Prior LFT increase and the number of concomitant medications were included in 


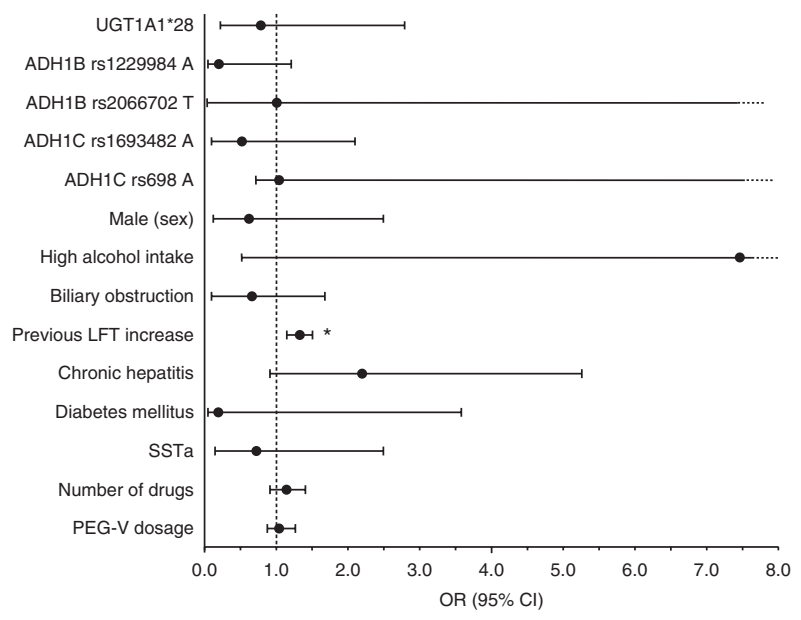

\section{Figure 1}

Backward stepwise logistic regression analysis of liver function test (LFT) increases during pegvisomant treatment in a group of 112 acromegalic patients ( $P$ for removal $\geq 0.10$ ). $Y$-axis: predictors and covariates. $X$-axis: odds ratio with $95 \% \mathrm{Cl}$. Nonsignificant predictors are shown with odds ratios taken from the step before their exclusion from the model. ${ }^{*} P<0.05$ at the final step of the backward stepwise regression.

the final model in GOT and gamma-GT analyses (GOT: $B=48.8 \pm 16.4, \quad P<0.01 \quad$ and $B=3.0 \pm 1.5, \quad P=0.04$; gamma-GT: $B=195.4 \pm 46.1, P<0.01$ and $B=9.9 \pm 4.1$, $P=0.02$ ). In GPT analysis, only prior LFT increase was a significant predictor $(B=57.5 \pm 19.7, P<0.01)$, together with the presence of diabetes mellitus ( $B=30.4 \pm 14.8, P=0.04)$. In all the analyses, no role of genes polymorphisms was found.

\section{Discussion}

PEG-V is a pegylated GH antagonist, which is a second choice therapeutic option in acromegaly, when surgery, SSTa, and radiotherapy do not control the disease $(1,2,3$, $4,5,6)$. Liver injury, is a serious adverse effect of PEG-V treatment, occurring in $5-10 \%$ of treated patients $(4,5,6)$. The underlying pathogenetic mechanisms of this phenomenon that may lead to treatment withdrawal are unclear. Both idiosyncratic reaction and biliary obstruction have been proposed (6). Recently, PEG-V hepatotoxicity has been linked to a common polymorphism (allele 28) of UGT1A1 gene (7) that is responsible for Gilbert's syndrome (8). As UGT1A1 is included in the liver detoxification system, this study suggested that this enzymatic network may be involved in the susceptibility to PEG-V. In fact, it has been demonstrated that polyethylene glycol is excreted intact by the biliary system and metabolized by liver, although this represents definitively a minor metabolization route compared with urine excretion (11). The biliary and liver metabolism of biological molecules associated with polyethylene glycol suggests a possible involvement of ADH enzymes in PEG-V metabolism. Interestingly, some $\mathrm{ADH} 1 \mathrm{~B}$ and $1 \mathrm{C}$ polymorphisms have been associated with alcohol pharmacokinetics, alcoholism, and alcoholic hepatopathy $(12,13$, $14,15,16)$.

On this basis, this study was carried out to evaluate whether polymorphic variants of UGT1A1 and $A D H$ genes may be associated with mild or severe liver disease in acromegalic patients during PEG-V treatment. In this multicenter study, UGT1A1*28, ADH1C rs1693482, ADH1C rs698, ADH1B rs1229984, and ADH1B rs2066702 polymorphisms were evaluated together with clinical, pharmacological, and biochemical data in 112 acromegalic patients with a long follow-up, from diagnosis to 3-108 months of treatment. Taking into account the rarity of the disorder under investigation, i.e. resistance to SSTa in unsuccessfully surgically treated acromegalic patients, the cohort of patients here reported can be regarded as a large series.

The global incidence of abnormal LFT at baseline was $6.3 \%$, a value consistent with that reported in the Caucasian population. Conversely, the prevalence of cholelithiasis in acromegalic patients was definitely high (40\%), probably due to previous SSTa treatment, which is known to have gallbladder stone formation as a common side effect. As expected, the UGT1A1*28 allele was significantly associated with Gilbert's syndrome as well as with higher bilirubin levels in acromegalic patients, while no significant difference in the allele distribution between patients and controls was detected. Similarly, no significant difference in ADH1C rs1693482, ADH1C rs698, ADH1B rs1229984, and ADH1B rs2066702 polymorphisms distribution in acromegalic patients and controls was observed.

This study did not confirm previous results, indicating that UGT1A1*28 genotype and male gender may predict an increased incidence of LFT abnormalities during PEG-V therapy in acromegaly (7). The only study so far available on this topic was carried out on a small series of patients under PEG-V therapy, i.e. 36 patients in comparison with the present series of 112 patients. Moreover, in that study the incidence of abnormal LFT during PEG-V therapy was higher in comparison with that observed in the present report and in previous observational studies. Both studies had similar protocols to obtain a complete liver panel in all follow-up visits and systematic records of LFT abnormalities, including mild and transient changes. 
The follow-up duration was different (17 months, IQR: $7-22$ vs $30 \pm 19$ months), although the timing of hepatotoxicity was similar. Moreover, it is tempting to speculate that the different susceptibilities to PEG-V-induced liver injury might be related to difference in genetic and environmental factors, together with drug interactions and concomitant diseases. A largest cohort of patients with longest follow-up should be screened to obtain an adequate statistical power, but this is a common issue in case of rare disease such as resistant acromegaly.

In analogy with the data obtained by investigating UGT1A1*28 genotype, no ADH variants were associated with liver toxicity induced by PEG-V. Admittedly, this study has some limitations, as we did not investigate other UGT1A1 and ADH variations in the coding, noncoding, or promoter regions. Moreover, we did not analyze the polymorphisms of UGT1A7*3 and UGT1A6*2 known to be linked to allele 28 and involved in drug glucuronidation $(19,20)$. Taken together, these results suggest a minor, if any, role of $U G T 1 A 1$ and $A D H$ gene polymorphisms on PEG-V-induced liver injury in acromegaly, although further studies are needed to fully clarify the UGT1A role in this particular drug toxicity.

This study points to a major impact of liver 'status' on the development of PEG-V-induced liver toxicity. In fact, linear and logistic regression analyses performed to analyze the whole set of factors which may interfere with liver function showed that prior episodes of LFT abnormalities in clinical history, the number of concomitant medications, and diabetes mellitus were associated with increased risk of hepatotoxicity. It is therefore tempting to hypothesize that potentially hepatotoxic drugs, taken by a relevant proportion of patients of the present cohort, might have a greater impact on hepatotoxicity development than PEG-V itself.

In this study, SSTa were administered in combination with PEG-V in about a half of patients to control residue regrowth, which is a possible outcome in patients receiving PEG-V due to the lack of GH feedback on somatotrophs. Interestingly, SSTa were not included among the drugs associated with increased risk of liver injury $(\mathrm{OR}=1.86$, 95\% CI $0.596-2.87, P=\mathrm{NS}$ ). These data are in agreement with previous studies investigating the long-term efficacy and safety of combined treatment of SSTa and PEG-V in acromegaly $(9,10)$. In fact, by investigating acromegalic patients who received PEG-V plus SSTa mild or overt LFT abnormalities were found in $\sim 25 \%$ of subjects, an incidence approaching that observed in this study. On the other hand, this study confirms a role of diabetes mellitus in PEG-V-induced LFT increase $(B=30.4 \pm 14.8$,
$P=0.04)$ as previously reported $(8,9)$, although no increase in hepatotoxicity risk (OR) was detected by logistic regression analysis. Moreover, as confirmed by previous studies $(3,21)$, no correlation between PEG-V dosage and liver toxicity was found.

In conclusion, no association between UGT1A1 and $\mathrm{ADH}$ polymorphisms and PEG-V hepatotoxicity was found in this study, although the low number of events together with multiple risk factors for liver toxicity (in particular hepatotoxic drugs) present in this series of patients did not allow definite conclusions. From a clinical point of view, prior episodes of LFT increase and the number of concomitant drugs taken by the patients seemed to have a major role in liver toxicity. This suggests that acromegalic patients treated with PEG-V and multiple other medical therapies should be carefully managed.

Supplementary data

This is linked to the online version of the paper at http://dx.doi.org/10.1530/ EJE-13-0657.

\section{Declaration of interest}

The authors declare that there is no conflict of interest that could be perceived as prejudicing the impartiality of the research reported.

\section{Funding}

This work was partially supported by Associazione Italiana per la Ricerca sul Cancro, Milan (grant number MFAG 8972 to G Mantovani); Progetti di Ricerca di Interesse Nazionale (PRIN) (grant number 2009YJTBAZ_004 to A G Lania); Ricerca Corrente Funds from the Fondazione Istituto di Ricerca e Cura a Carattere Scientifico (IRCCS) Ca' Granda-Milan (grant number 180-02 to A Spada); and Pfizer Italia.

\section{Acknowledgements}

The authors thank Dr Corinna Giuliani.

\section{References}

1 Kopchick JJ, Parkinson C, Stevens EC \& Trainer PJ. Growth hormone receptor antagonists: discovery, development, and use in patients with acromegaly. Endocrine Reviews 200223 623-646. (doi:10.1210/ er.2001-0022)

2 Colao A, Pivonello R, Auriemma RS, De Martino MC, Bidlingmaier M, Briganti F, Tortora F, Burman P, Kourides IA, Strasburger CJ et al. Efficacy of 12-month treatment with the GH receptor antagonist pegvisomant in patients with acromegaly resistant to long-term, highdose somatostatin analog treatment: effect on IGF-I levels, tumor mass, hypertension and glucose tolerance. European Journal of Endocrinology 2006154 467-477. (doi:10.1530/eje.1.02112)

3 Schreiber I, Buchfelder M, Droste M, Forssmann K, Mann K, Saller B \& Strasburger CJ \& German Pegvisomant Investigators. Treatment of 
acromegaly with the GH receptor antagonist pegvisomant in clinical practice: safety and efficacy evaluation from the German Pegvisomant Observational Study. European Journal of Endocrinology 2007156 75-82. (doi:10.1530/eje.1.02312)

4 Trainer PJ, Drake WM, Katznelson L, Freda PU, Herman-Bonert V, van der Lely AJ, Dimaraki EV, Stewart PM, Friend KE, Vance ML et al. Treatment of acromegaly with the growth hormone-receptor antagonist pegvisomant. New England Journal of Medicine 2000342 1171-1177. (doi:10.1056/NEJM200004203421604)

5 Sherlock M, Woods C \& Sheppard MC. Medical therapy in acromegaly. Nature Reviews. Endocrinology 20117 291-300. (doi:10.1038/nrendo. 2011.42)

6 Hodish I \& Barkan A. Long-term effects of pegvisomant in patients with acromegaly. Nature Clinical Practice. Endocrinology \& Metabolism 20084 324-332. (doi:10.1038/ncpendmet0831)

7 Bernabeu I, Marazuela M, Lucas T, Loidi L, Alvarez-Escolá C, Luque-Ramírez M, Fernandez-Rodriguez E, Paniagua AE, Quinteiro C \& Casanueva FF. Pegvisomant-induced liver injury is related to the UGT1A1*28 polymorphism of Gilbert's syndrome. Journal of Clinical Endocrinology and Metabolism 201095 2147-2154. (doi:10.1210/jc. 2009-2547)

8 Ehmer U, Kalthoff S, Fakundiny B, Pabst B, Freiberg N, Naumann R, Manns MP \& Strassburg CP. Gilbert syndrome redefined: a complex genetic haplotype influences the regulation of glucuronidation. Hepatology 201255 1912-1921. (doi:10.1002/hep.25561)

9 Neggers SJ, van Aken MO, Janssen JA, Feelders RA, de Herder WW \& van der Lely AJ. Long-term efficacy and safety of combined treatment of somatostatin analogs and pegvisomant in acromegaly. Journal of Clinical Endocrinology and Metabolism 200792 4598-4601. (doi:10.1210/ jc.2007-1234)

10 Neggers SJ, de Herder WW, Janssen JA, Feelders RA \& van der Lely AJ. Combined treatment for acromegaly with long-acting somatostatin analogs and pegvisomant: long-term safety for up to 4.5 years (median 2.2 years) of follow-up in 86 patients. European Journal of Endocrinology 2009160 529-533. (doi:10.1530/EJE-08-0843)

11 Webster R, Didier E, Harris P, Siegel N, Stadler J, Tilbury L \& Smith D. PEGylated proteins: evaluation of their safety in the absence of definitive metabolism studies. Drug Metabolism and Disposition 200735 9-16. (doi:10.1124/dmd.106.012419)

12 Mulligan CJ, Robin RW, Osier MV, Sambuughin N, Goldfarb LG, Kittles RA, Hesselbrock D, Goldman D \& Long JC. Allelic variation at alcohol metabolism genes (ADH1B, ADH1C, ALDH2) and alcohol dependence in an American Indian population. Human Genetics 2003 113 325-336. (doi:10.1007/s00439-003-0971-z)
13 Tolstrup JS, Nordestgaard BG, Rasmussen S, Tybjaerg-Hansen A \& Grønbaek M. Alcoholism and alcohol drinking habits predicted from alcohol dehydrogenase genes. Pharmacogenomics Journal $2008 \mathbf{8}$ 220-227. (doi:10.1038/sj.tpj.6500471)

14 Tóth R, Fiatal S, Petrovski B, McKee M \& Adány R. Combined effect of ADH1B RS1229984, RS2066702 and ADH1C RS1693482/ RS698 alleles on alcoholism and chronic liver diseases. Disease Markers 201131 267-277. (doi:10.1155/2011/350528)

15 Martínez C, Galván S, Garcia-Martin E, Ramos MI, Gutiérrez-Martín Y \& Agúndez JA. Variability in ethanol biodisposition in whites is modulated by polymorphisms in the ADH1B and ADH1C genes. Hepatology 201051 491-500. (doi:10.1002/hep.23341)

16 Kimura M \& Higuchi S. Genetics of alcohol dependence. Psychiatry and Clinical Neurosciences 201165 213-225. Review. (doi:10.1111/ j.1440-1819.2011.02190.x)

17 Filopanti M, Olgiati L, Mantovani G, Corbetta S, Arosio M, Gasco V, De Marinis L, Martini C, Bogazzi F, Cannavò S et al. Growth hormone receptor variants and response to pegvisomant in monotherapy or in combination with somatostatin analogs in acromegalic patients: a multicenter study. Journal of Clinical Endocrinology and Metabolism 2012 97 E165-E172. (doi:10.1210/jc.2011-1769)

18 Filopanti M, Barbieri AM, Angioni AR, Colao A, Gasco V, Grottoli S, Peri A, Baglioni S, Fustini MF, Pigliaru F et al. Dopamine D2 receptor gene polymorphisms and response to cabergoline therapy in patients with prolactin-secreting pituitary adenomas. Pharmacogenomics Journal 20088 357-363. (doi:10.1038/tpj.2008.1)

19 Köhle C, Möhrle B, Münzel PA, Schwab M, Wernet D, Badary OA \& Bock KW. Frequent co-occurrence of the TATA box mutation associated with Gilbert's syndrome (UGT1A1*28) with other polymorphisms of the UDP-glucuronosyltransferase-1 locus (UGT1A6*2 and UGT1A7*3) in Caucasians and Egyptians. Biochemical Pharmacology 200365 1521-1527. (doi:10.1016/ S0006-2952(03)00074-1)

20 Peters WH, te Morsche RH \& Roelofs HM. Combined polymorphisms in UDP-glucuronosyltransferases 1A1 and 1A6: implications for patients with Gilbert's syndrome. Journal of Hepatology 200338 3-8. (doi:10.1016/S0168-8278(02)00306-9)

21 Biering H, Saller B, Bauditz J, Pirlich M, Rudolph B, Johne A, Buchfelder M, Mann K, Droste M, Schreiber I et al. Elevated transaminases during medical treatment of acromegaly: a review of the German pegvisomant surveillance experience and a report of a patient with histologically proven chronic mild active hepatitis. European Journal of Endocrinology 2006154 213-220. (doi:10.1530/ eje.1.02079)

Received 9 August 2013

Revised version received 8 November 2013

Accepted 11 November 2013 